\title{
Technical note: A deformable phantom for dynamic modeling in radiation therapy
}

\author{
Rojano Kashani, ${ }^{\text {a) }}$ Kwok Lam, Dale Litzenberg, and James Balter \\ Department of Radiation Oncology, University of Michigan, Ann Arbor, \\ Michigan 48109
}

(Received 7 July 2006; revised 9 August 2006; accepted for publication 20 October 2006; published 20 December 2006)

\begin{abstract}
A deformable phantom was developed to aid in quality assurance for dynamic imaging and targeting techniques in radiation therapy. Made of simple materials combined with standard components for imaging and motion experiments, this phantom can be relatively easily constructed and used for both diagnostic imaging and dosimetry. Repeat imaging studies indicate that the phantom meets criteria of relative attenuation, deformation, and reproducibility of configuration, necessary for quality assurance of radiographic and tomographic targeting. () 2007 American Association of Physicists in Medicine. [DOI: 10.1118/1.2400612]
\end{abstract}

Key words: 4D computed tomography, 4D phantom, organ motion, deformation

\section{INTRODUCTION}

The vast array of research and commercial projects aimed at measuring and incorporating short-term movement in treatment planning and delivery demand sufficient quantitative means of verification. ${ }^{1-6}$ While analytic models and patient data are useful, a reproducible, compartmented, mechanical phantom is critical to proper commissioning and use of new systems for physiological motion management in radiation therapy.

\section{A. Phantom components}

A diagnostic thoracic phantom (RS-330, Radiology Support Devices, Long Beach, CA) provided the main shell of the phantom. This phantom has a skeleton, as well as a lungequivalent insert, with additional tissue-mimicking sections for a mediastinum and various tumor-simulating nodules. An insert for an abdominal cavity, composed of a uniform density material, was removed. In its place, an extension of the existing lung insert was created using high density foam (Fig. 1). This process created a section of the phantom with a rigid skeleton and tissue wall, surrounding a compressible internal cavity.

The insert was evaluated for relative attenuation (effective attenuation relative to water) using a commercial computed tomography (CT) scanner (HighSpeed, General Electric, Milwaukee WI). Scans demonstrated a relative attenuation of $5 \%-10 \%$ of water. To mimic lung attenuation for diagnostic $\mathrm{kV}$ imaging, the foam insert was infused with iodine. This was accomplished by soaking the insert in a solution of iodinated contrast agent (Ultravist 300, Berlex Laboratories, Montville NJ) diluted in water in an approximately 5:1 (water:ultravist) mix. After infusion, the foam was allowed to dry. Subsequent scanning demonstrated Hounsefield Units of -800 to -600 , more consistent with lung attenuation as reported by previous investigators. ${ }^{7}$

Tumor-simulating inserts of varying density and size were embedded in the foam. To facilitate initial studies focusing on imaging and geometric localization, the structures selected were rigid objects of known shape (balls) and various compositions (hard rubber, plastic, porous foam) to generate varying electron densities. The objects were inserted at various locations by small incisions in the foam, and further held in place with glue.

An actuator-driven diaphragm was created to compress/ decompress the foam according to previously defined arbitrary breathing profiles. A single-axis actuator, previously designed for motion experiments and similar to systems described by other investigators, ${ }^{8-11}$ was employed. The "diaphragm" was created by cutting a 1-cm-thick sheet of Lucite to a size slightly smaller than the cavity into which the foam insert was placed. This diaphragm was attached to the driving rod of the actuator via a wooden brace, with a three-point attachment to distribute the force of the actuator uniformly across the Lucite plate (Fig. 2). The phantom was braced cranially to limit the possible rigid body movement of the whole system under compression and relaxation.

The actuator system has a control software environment that permits customized positioning, and complex temporal motion profiles to be programmed and executed. Breathing patterns were programmed that represented periodic functions, ${ }^{12}$ variations on periodicity and amplitude to simulate known but irregular breathing, and finally measured position/amplitude variations from patients studied under a review board-approved protocol using an external monitoring system (RPM, Varian, Palo Alto CA). Although significantly larger motions could be programmed, the tests performed used diaphragm compressions of up to $3 \mathrm{~cm}(20 \%$ volume reduction of the compressible cavity). 


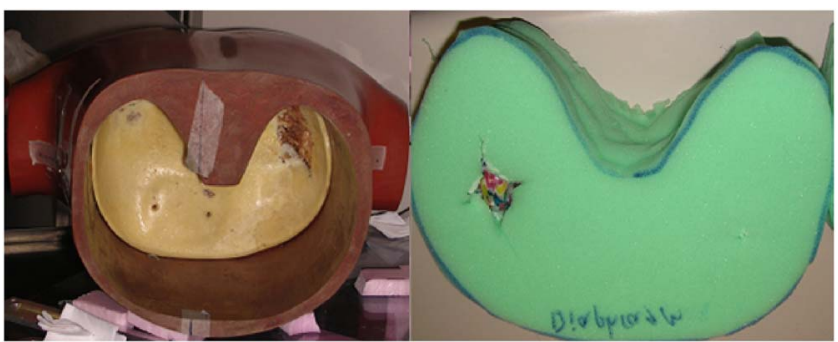

FIG. 1. The diagnostic phantom with the abdominal insert removed (left), the high density foam insert (right).

\section{SYSTEM PERFORMANCE}

\section{A. Relative attenuation}

The relative attenuation (based on Hounsfield Units) in the foam during the "inhale" (uncompressed) state had a mean value of 0.177 with a standard deviation of 0.143 indicating a large variation in attenuation between voxels. Under $3 \mathrm{~cm}$ of compression, these values changed to a mean of 0.196 and standard deviation of 0.139 . An example from patient data acquired under a review board-approved protocol, with similar diaphragm movement (approximately $3 \mathrm{~cm}+/-3 \mathrm{~mm}$ ) showed a change in lung volume of $17 \%$ and a change in density from 0.202 at inhale to 0.244 at exhale.

\section{B. Motion under compression}

Important characteristics of this phantom include differential motion of various points under compression, as well as reproducible configuration at the same amount of compression (breathing state). To evaluate reproducibility, CT scans were taken of the phantom under various states of compression (Fig. 3). These scans were repeated in the same session, as well as in a separate session 3 months later to evaluate long-term reproducibility of position at a given compression state.

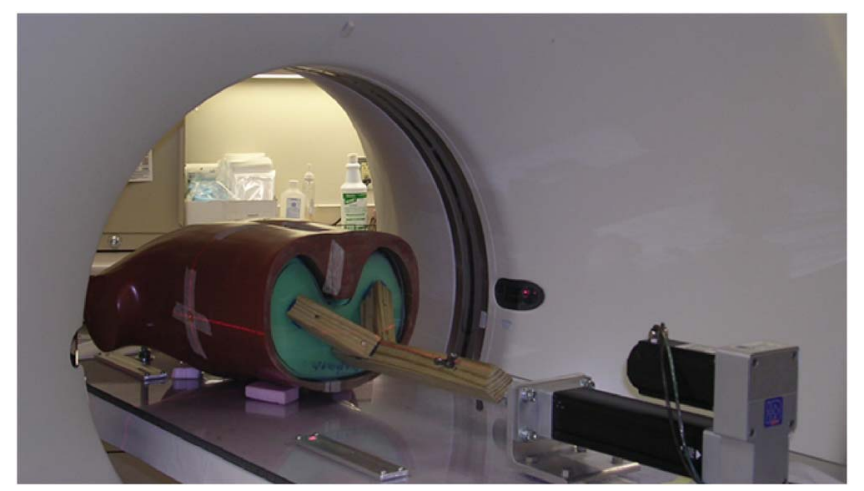

FIG. 2. The setup of the phantom and motion actuator on the CT scanner.

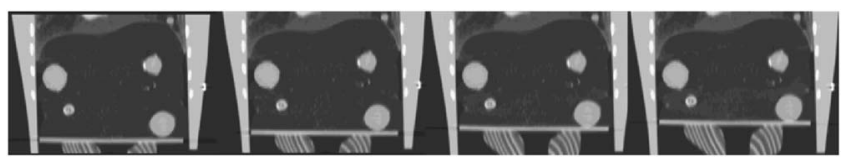

FIG. 3. CT scan of the phantom with the foam and tumor simulating inserts under compressions (diaphragm movements) of $0,1,2$, and $3 \mathrm{~cm}$ (from left to right).

Table I shows the reproducibility of tumor positions under repeat compressions. Intermediate tumor positions are reproducible to within $1.3 \mathrm{~mm}$. Table II shows the differential movement of targets in various locations within the phantom. As expected, targets closer to the diaphragm moved farther than those more cranially positioned. Compressions of over $50 \%$ of the foam insert have been demonstrated, with regional target motion ranging from $95 \%$ of the actuator motion at the diaphragm to $<30 \%$ near the "apex" (the interface with the existing lung insert).

Repeat reproducibility studies performed 3 months after the first study indicated that the relative local reproducibility stays the same (mean of zero and standard deviation of less than $1.1 \mathrm{~mm}$ ).

\section{CONCLUSION}

This phantom is simple, efficient, and viable. Experiments in quantitative dynamic modeling of breathing-induced deformations are under way using this system. The reproducibility of the deformation induced in the phantom will allow the use of this phantom in validation of image-based patient modeling and targeting, as well as analytic models and estimation methods that incorporate breathing motion into dose calculation. Ongoing work involves addition of dosimeters to this phantom, which will give us direct measurements for quality assurance of dose calculations incorporating breathing motion.

TABLE I. Reproducibility of tumor position between repeat compressions. For each diaphragm compression, the position of the center of the tumor in the first scan was taken as the reference and compared to the second scan. The results presented here include all seven tumors and all compressions.

\begin{tabular}{lccc}
\hline \hline & Right/Left & $\begin{array}{c}\text { Anterior/ } \\
\text { Posterior }\end{array}$ & $\begin{array}{c}\text { Superior/ } \\
\text { Inferior }\end{array}$ \\
\hline $\begin{array}{l}\text { Average difference between } \\
\text { multiple compressions (cm) }\end{array}$ & -0.03 & -0.02 & -0.02 \\
Standard deviation (cm) & 0.06 & 0.05 & 0.13 \\
Maximum deviation (cm) & 0.13 & 0.08 & 0.29 \\
\hline \hline
\end{tabular}


TABLE II. Motion of tumor simulating inserts under different diaphragm compressions. For each diaphragm compression (shown at the top), the actual motion in centimeters and the motion as a percentage of the diaphragm compression are shown. The error in each measurement is also shown in parentheses for both values.

\begin{tabular}{|c|c|c|c|c|c|c|c|c|c|c|c|c|}
\hline \multirow{3}{*}{$\begin{array}{l}\text { Distance to } \\
\text { diaphragm } \\
(\mathrm{cm})\end{array}$} & \multicolumn{4}{|c|}{$\begin{array}{l}\text { Compression }=1.2 \mathrm{~cm} \\
\text { Tumor motion (error) }\end{array}$} & \multicolumn{4}{|c|}{$\begin{array}{l}\text { Compression }=2.1 \mathrm{~cm} \\
\text { Tumor motion (error) }\end{array}$} & \multicolumn{4}{|c|}{$\begin{array}{l}\text { Compression }=3.0 \mathrm{~cm} \\
\text { Tumor motion (error) }\end{array}$} \\
\hline & \multicolumn{2}{|c|}{$\mathrm{cm}$} & \multicolumn{2}{|c|}{$\%$} & \multicolumn{2}{|c|}{$\mathrm{cm}$} & \multicolumn{2}{|c|}{$\%$} & \multicolumn{2}{|c|}{$\mathrm{cm}$} & \multicolumn{2}{|c|}{$\%$} \\
\hline & 1.01 & $(0.05)$ & 84 & (4.2) & 1.89 & $(0.04)$ & 90 & (1.9) & 2.85 & $(0.10)$ & 95 & (3.3) \\
\hline 2.05 & 0.85 & $(0.09)$ & 71 & (7.5) & 1.69 & $(0.10)$ & 80 & $(4.8)$ & 2.53 & (0.06) & 84 & (2.0) \\
\hline 4.33 & 0.55 & $(0.10)$ & 46 & (8.3) & 1.24 & $(0.12)$ & 59 & (5.7) & 1.87 & $(0.15)$ & 62 & (5.0) \\
\hline 8.53 & 0.30 & $(0.07)$ & 25 & (5.8) & 0.60 & $(0.10)$ & 29 & $(4.8)$ & 0.90 & $(0.15)$ & 30 & $(5.0)$ \\
\hline 8.77 & 0.22 & $(0.23)$ & 18 & (19) & 0.53 & $(0.07)$ & 25 & (3.3) & 0.83 & (0.04) & 28 & (1.3) \\
\hline 10.55 & 0.24 & (0.16) & 20 & (13) & 0.45 & $(0.15)$ & 21 & (7.1) & 0.84 & $(0.06)$ & 28 & (2.0) \\
\hline 10.63 & 0.25 & $(0.35)$ & 21 & (29) & 0.37 & $(0.16)$ & 18 & (7.6) & 0.76 & $(0.06)$ & 25 & $(2.0)$ \\
\hline
\end{tabular}

\section{ACKNOWLEDGMENT}

This work was supported by NIH P01-CA59827.

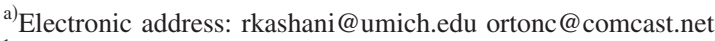
${ }^{1}$ P. J. Keall, J. V. Siebers, S. Joshi, and R. Mohan, "Monte Carlo as a four-dimensional radiotherapy treatment planning tool to account for respiratory motion," Phys. Med. Biol. 49(16), 3639-3648 (2004).

${ }^{2}$ E. Rietzel, G. T. Chen, N. C. Choi, and C. G. Willet, "Four-dimensional image-based treatment planning: Target volume segmentation and dose calculation in the presence of respiratory motion," Int. J. Radiat. Oncol., Biol., Phys. 61(5), 1535-1550 (2005).

${ }^{3}$ P. J. Keall, S. Joshi, S. S. Vedam, J. V. Siebers, V. R. Kini, and R. Mohan, "Four-dimensional radiotherapy planning for DMLC-based respiratory motion tracking," Med. Phys. 32(4), 942-951 (2005).

${ }^{4}$ C. Thilmann, P. Haring, L. Thilmann, J. Unkelbach, B. Rhein, S. Nill, P. Huber, E. Janisch, C. Thieke, and J. Debus, "The influence of breathing motion on intensity modulated radiotherapy in the step-and-shoot technique: Phantom measurements for irradiation of superficial target volumes," Phys. Med. Biol. 51(6), 117-26 (2006).

${ }^{5}$ S. Flampouri, S. B. Jiang, G. C. Sharp, J. Wolfgang, A. A. Patel, and N. C. Choi, "Estimation of the delivered patient dose in lung IMRT treatment based on deformable registration of 4D-CT data and Monte Carlo simulations," Phys. Med. Biol. 51(11), 2763-2779 (2006).
${ }^{6}$ B. C. Cho and T. Craig, "More optimal dose distributions for moving lung tumours: A planning study," Radiother. Oncol. 79(1), 122-130 (2006).

${ }^{7}$ R. M. Henkelman and K. Mah, "How important is breathing in radiation therapy for the thorax?," Int. J. Radiat. Oncol., Biol., Phys. 8(11), 20052010 (1982).

${ }^{8}$ F. Banovac, J. Tang, S. Xu, D. Lindisch, H. Y. Chung, E. B. Levy, T. Chang, M. F. McCullough, Z. Yaniv, B. J. Wood, and K. Cleary, "Precision targeting of liver lesions using a novel electromagnetic navigation device in physiologic phantom and swine," Med. Phys. 32(8), 2698-2705 (2005).

${ }^{9}$ S. A. Naqvi and W. D. D'Souza, “A stochastic convolution/superposition method with isocenter sampling to evaluate intrafraction motion effects in IMRT," Med. Phys. 32(4), 1156-63 (2005).

${ }^{10}$ W. Lu, P. J. Parikh, J. P. Hubenschmidt, D. G. Politte, B. R. Whiting, J. D. Bradley, S. Mutic, and D. A. Low, "Reduction of motion blurring artifacts using respiratory gated CT in sonogram space: A quantitative evaluation," Med. Phys. 32(11), 3295-304 (2005).

${ }^{11}$ M. J. Fitzpatrick, G. Starkschall, P. Balter, J. A. Antolak, T. Guerrero, C. Nelson, P. Keall, and R. Mohan, "A novel platform simulating irregular motion to enhance assessment of respiration-correlated radiation therapy procedures,” J. Appl. Clin. Med. Phys. 6(1), 13-21 (2005).

${ }^{12}$ A. E. Lujan, E. W. Larsen, J. M. Balter, and R. K. Ten Haken, "A method for incorporating organ motion due to breathing into $3 \mathrm{D}$ dose calculations," Med. Phys. 26(5), 715-720 (1999). 